\title{
A Bayesian Method for Sequential Sampling and Forecasting in Agricultural Pest Management*
}

\author{
Richard E. Plant' and L. T. Wilson ${ }^{2}$ \\ Departments of Mathematics' and Entomology², University of California, \\ Davis, California 95616, U.S.A.
}

\begin{abstract}
SUMMARY
We present a method for sequential estimation of the state of infestation of an agricultural crop by a pest arthropod. The method, which is based on Bayesian statistical theory, establishes stopping curves by requiring that a confidence interval be placed entirely on one side or the other of an economic threshold. The results of a given sequential sample are used to modify the prior of the subsequent sample by assuming that the infestation follows a particular growth law. The parameters of this growth law are themselves uncertain and are described by subjective distributions that are modified by the sampling. A major advantage of our method is that it readily admits the incorporation of a forecasting scheme. After describing the development of the method in general terms, we give a specific example of the application of the method to the sampling of spider mites on cotton in the San Joaquin Valley, California.
\end{abstract}

\section{Introduction}

One of the fundamental concepts of integrated pest management (IPM) in agriculture is that of the economic threshold. This may be defined in various ways, but basically it represents the state of infestation of a particular crop by a particular pest at which intervention to control that pest becomes economicallyjustified (Stem et al., 1959).Control measures should not be applied unless the infestation reaches the economic threshold. It is evident that if this concept is to have practical applicability, then the grower must be provided with sampling techniques that permit the accurate assessment of the state of the infestation. Depending on the crop and the pest, this state is generally defined either as the number of individuals per sampling unit, or as the proportion of infested units. The application that concerns us most is spider mites (Tetranychus spp.) as a pest of cotton in the San Joaquin Valley, California.

Spider mites are capable of overwintering as adult females in the soil of fields infested the previous summer, as well as in soil leaf litter and under the bark of surrounding perennial crops such as almonds and grapes. Excessive early season use of pesticides for control of other cotton pests often disrupts spider mite predators. When combined with warm temperatures, this disruption can allow the spider mite population density to reach $8 \times 10^{8} /$ ha. in a matter of weeks (Wilson, unpublished data). Field experiments conducted over the last few years indicate that in extreme cases yield reductions as high as $70 \%$ may occur, resulting in a potential revenue loss of approximately $\$ 1600 /$ ha. The explosive damage potential of spider mites has led to prophylactic acaricide treatments, and presently spider mites are the most treated cotton pest in California. Problems with pesticide resistance and potential disruption of natural enemies of other pests, however, warrant an economic threshold-based approach to spider mite management.

\footnotetext{
${ }^{*}$ Research supported by NSF Grant MCS 81-21413 and by a USDA C/IPM Grant.
}

Key words: Bayesian sampling; Pest management; Sequential estimation. 
Since spider mites are so small and abundant, it is impractical to count them, so the sampler records the proportion of leaves with one or more mites (Wilson et al., 1983). Sequential sampling methods are appropriate for this application, particularly for row crops. Several papers have been written in which Wald's (1947) sequential probability ratio test (SPRT) is applied to agricultural or silvicultural pest management problems (e.g., Waters, 1955; Sylvester and Cox, 1961; Allen, Gonzalez, and Gokhale, 1972; Onsager, 1976; Sterling, 1976). As Wetherill (1966) points out, however, the SPRT is perhaps not the most effective use of the data for this particular problem. One criticism is that the fixing of the upper and lower thresholds is often completely arbitrary.

A second, more fundamental criticism deals with the type of distribution used in the SPRT. In counting (as opposed to presence-absence sampling), the insect population is most commonly described by a negative binomial distribution. However, the value of the overdispersion coefficient $k$ depends on the mean population size $m$. Since $m$ is the parameter of interest in the sampling, one is forced to make an assumption about the value of $k$, based perhaps on previous data or on values in the literature. The present article, however, focuses on presence-absence sampling, for which no such assumptions are necessary.

A third criticism of the SPRT in this application, at least in its usual form, is that it does not permit the sampler to take into account data external to the current sample. For example, in the case of spider mites on cotton, a sampler (hereafter called the scout) may sample periodically, as many as ten or more times, before taking any control actions. If the growth rate of the pest population is known, or can be estimated, then the data accumulated from previous samples is relevant to the current one. In addition, data concerning weather patterns, predator population estimates, and so forth, may be available. If so, these data would be relevant to estimates of the pest population level, and to forecasts of its future growth.

A number of writers, including Wetherill (1966), have suggested that the problem might better be phrased in the context of estimation rather than hypothesis testing. Anscombe (1949)developed a sequential estimation procedure, later rederived independently by Kuno (1969), and attempts have been made to apply this to agricultural problems (e.g., Allen et al., 1972). This method, however, does not take the economic threshold into account and thus is not able to adjust the accuracy of the estimate for the distance from this threshold. More recently, Wilson (1982) has developed a sequential estimation method based on the establishment of a confidence interval on either side of the economic threshold. This method, which is similar to one of Iwao (1975), has worked quite well in several applications (e.g., Wilson and Room, 1983; Zalom et al., 1983). For counting applications, Wilson's method uses Taylor's (196I) variance-mean formula, and thus does not fall prey to the problem of having to assume a value of $k$.

Wilson's method is, however, still subject to the third criticism raised above; it does not permit the incorporation of relevant data external to the sample. In this paper we formulate a sequential estimation procedure that is intended to address this point. The usual way to incorporate previously obtained data into an estimation problem is by Bayesian statistical methods, and these are the methods we use. As such, we leave ourselves open to the second criticism above, namely, we are forced to assume a particular distribution. Since our primary concern is with the presence-absence sampling of spider mites, however, we may assume that the sampling units are binomially distributed, so that we do not encounter the problem of determining the overdispersion coefficient.

There are several practical constraints that must be met in the development of a sampling scheme intended for agricultural field application. One is that the sampling protocol must be designed so that sampling may be carried out by individuals who have no statistical training whatsoever, and thus should be as simple as possible. A second is that all the 
information needed by the scout must be capable of being placed on a card that can be easily carried in the field. A third constraint is that if the method is to be given the best possible chance of being adopted, it should be similar to methods that are already in general use.

Our method, described in the next section, is designed with these constraints in mind. It differs from traditional Bayesian sequential sampling methods (e.g., Berger, 1980) in that we do not take unit costs into account, and we do not use a dynamic programming formulation. Rather, following Wilson (1982), we continue sampling until a Bayesian confidence interval with given error rates may be placed entirely on one side or the other of the economic threshold. This is possible in principle since with probability 1 the true population level is either above or below the economic threshold at any particular time. Our approach may also be justified partly on the grounds of meeting the three constraints described above (particularly the second two). Additional justification is that the unit costs of the sampling are considerably less important than the cost of making an incorrect decision. Indeed, it is likely that the scout, to be on the safe side, may continue to sample beyond the point where he theoretically could stop. Our method is designed to make use of the additional information that this extra sampling provides.

To convert the posterior distribution of one sampling sequence into the prior of the next we assume that the parameter representing the level of infestation changes in a way whose dynamics depend on parameters that are themselves unknown. At each stage after the first we update our estimate of this growth rate parameter. We then forecast the future growth of the infestation. This is done to convert the posterior probability density for one sample into the prior density of the next. It is also used to forecast future growth of the infestation over time. This important feature allows the grower to better determine the time at which intervention may become necessary, allowing him to schedule other actions such as irrigation and fertilization. If the level of infestation is near the economic threshold the forecast may also be used to determine when to take the next sample.

The development of the method in the next section is phrased in terms of general prior and likelihood densities. In $\mathbf{9 3}$ we give a specific example of how the method is applied to the problem of presence-absence sampling of spider mites on cotton. We make use of a binomial likelihood and for convenience we use a beta family of priors. We assume, based on field data, that the proportion of infested leaves follows a logistic growth law, and we assume a normal distribution for the unknown growth rate. The use of other densities and growth dynamics would be straightforward. In 94 we give a brief discussion of our results.

\section{Development of the Method}

In this section we derive the formulas of the sequential analysis procedure. First, we give a brief verbal description. Each individual sample is taken in the usual manner of sequential sampling. The scout walks along the crop row and periodically observes a sampling unit. In row crops, when sampling for mites, this unit typically is a single leaf, and for conciseness we shall refer to the unit as a leaf. For the ith leaf, the scout records the data $x_{i}$ [e.g., presence $(\mathbf{x},=1)$ or absence $\left(x_{i}=0\right)$ of the species in question]. Letting $n$ denote the number of leaves sampled so far, and letting $z_{n}=\sum_{i=1}^{n} \mathbf{x}$, the scout then compares $z_{n}$ with two values on a card, denoted $u_{n}$ and $l_{n}$. The loci of these values are called the stopping curves. If $z_{n} \geq u_{n}$, then sampling is stopped and the infestation is judged to be above the economic threshold. If $z_{n} \leqslant l_{n}$, then sampling is stopped and the infestation is judged to be below the economic threshold. If $l_{n}<z_{n}<u_{n}$, then another sample is taken.

Assume that a sample is taken at time $t=0$, where $t$ is measured in degree-days [see Wilson and Bamett (1983) for an explanation of this term]. Let $T(t)$ denote the economic threshold at time $t$, and let $\theta(t)$ denote the true value of the parameter (e.g., proportion of 
infested leaves) at this time. For conciseness we shall often suppress the arguments of these functions. Let $\alpha$ be the acceptable probability of deciding to apply the pesticide when the population is below the economic threshold, and let $\beta$ be the acceptable probability of deciding not to apply the pesticide when the population is above the economic threshold. For each $n$ the value of $l_{n}$ is obtained by requiring that $\operatorname{Pr}\left(\theta>T \mid I_{n}\right)$ be less than $\alpha$, where $I_{n}$ is the information state at the $n$th leaf, consisting of the values of $n$ and $z_{n}$. Similarly, the value of $u_{n}$ is obtained by requiring that $\operatorname{Pr}\left(\theta<T \mid I_{n}\right)$ be less than $\beta$. Let $\pi_{l}(\theta \mid I$,, $)$ denote the (posterior) probability density on $\theta(t)$ given $I_{n}$. Let $P(n, \mathrm{z}$, ) be defined by

$$
P(n, \mathrm{z},) \equiv \operatorname{Pr}\left(\theta<T \mid I_{n}\right)=\int_{0}^{T} \pi_{l}\left(\theta \mid I_{n}\right) d e .
$$

The equations defining $l_{n}$ and $u_{n}$ are therefore

$$
\begin{aligned}
& l_{n}=\max _{z_{n}}\left[z_{n} \mid 1-P(n, \mathrm{z},) \leqslant \mathrm{a}\right] ; \\
& u_{n}=\min _{z_{n}}\left[z_{n} \mid P(n, z,,) \leqslant \beta\right] .
\end{aligned}
$$

Given a prior probability density $\pi_{l}(\theta)$, the posterior density is obtained from Bayes formula as

$$
\pi_{l}\left(\theta \mid I_{n}\right)=\frac{\ell\left(\theta \mid I_{n}\right) \pi_{t}(\theta)}{m_{t}\left(I_{n}\right)}
$$

where $\ell\left(\theta \mid I\right.$, , ) and $m_{t}\left(I_{n}\right)$ are the likelihood and marginal densities, respectively.

Once the sampling is completed at time 0 by using the above procedure with $t=0$, the next step is to compute the prior $\pi_{l}(\theta)$ at the next planned sampling time. Assume that the parameter $\theta(t)$ obeys a dynamic relation of the form $\theta(t)=f[\theta(0) ; r, t]$, where $r$ is a growth rate parameter that is fixed, but not known with certainty, for a given season and location. In general, $r$ will depend on factors such as the number of predators present, the nutrient quality of the plant, and so forth. In principle, the value of $r$ may even be negative. Let $\rho(r)$ be a (prior) probability with density on $r$. Let $\phi_{t}(\theta ; r)$ be the inverse function of $f$, i.e., $\theta(0)=\phi_{l}\{f[\theta(0) ; r, t] ; r\}$. Let

$$
\left.\psi_{t}(\theta \mid r)=\pi_{0}\left[\phi_{t}(\theta ; r)\right] I_{n}\right](d \phi / d \theta)
$$

Then the prior density on $\theta$ at time $t$ is the expected value over $r$ of $\psi_{t}(\theta \mid r)$, namely,

$$
\pi_{l}(\theta)=\int_{-\infty}^{\infty} \psi_{l}(\theta \mid r) \rho(r) d r
$$

Having obtained this prior density, one may compute the stopping curves $l_{n}$ and $u_{n}$ at time $t$ using equations (2.1) and (2.2).

Equation (2.5) may also be used to forecast the state of infestation at any future time. The density $\pi_{t}(\theta)$ represents the subjective probability density of the state of infestation at that time $t$. Thus, for example, the expected value of the state of infestation at any future time, given present knowledge, may be computed by taking an expectation over $\boldsymbol{\theta}$ of this function. It is perhaps more appropriate to compute percentiles of the distribution. The pth percentile is the value $\tau$ satisfying

$$
p=\int_{0}^{r} \pi_{l}(\theta) d \theta
$$

Finally, after the sample is obtained at time $t$, the probability density $\rho(r)$ may be updated via Bayes' theorem. The likelihood function in this case is simply the density $\psi_{t}(\theta \mid r)$, computed in equation (2.4). Therefore, the posterior density on the growth rate $\boldsymbol{r}$ is given 
by

$$
\rho(r \mid I)=\frac{\psi_{t}\left(\theta_{l} \mid r\right) \rho(r)}{\pi\left(\theta_{l}\right)}
$$

where $I$ represents the values of $n$ and $z_{n}$ obtained in the sample at time $t$, and $\theta_{l}$ is the point estimate of 0 based on this information. The value of $\theta_{I}$ is $z_{n} / n$.

Having computed the relevant quantities at time $t$, one may then set the current time to 0 and repeat the process, proceeding in this way throughout the season. At each stage, one first obtains the upper and lower stopping curves. Then, based on the actual sample obtained, one may update the subjective density on the growth rate and project into the future. Based on these projections, one may establish the next appropriate time to take a sample, and one may also schedule other operations such as imgation and fertilization.

In the next section, we give a specific example of how our method would be applied. This example concerns binomial sampling and its application to the estimation of the state of infestation of spider mites (Tetranychus spp.) on cotton in the San Joaquin Valley of California.

\section{Application to Binomial Sampling}

The spider mite complex is a major pest of cotton in the San Joaquin Valley of California. It provides a particular challenge to the design of a pest management scheme both because mites are so small that they are difficult to see, and because they are capable of explosive growth in population. Many growers apply acaricide prophylactically to control spider mites. There is evidence, however, that a large portion of these applications are wasted. Therefore, a good method of sampling for mites, which must be a binomial sampling scheme since it is impractical to count them, may provide a way to reduce significantly the quantity of pesticide applied to cotton where mites are a major pest. Preliminary results (Wilson et al., 1983) indicate that Wilson's (1982) method works well. Thus, we are motivated to consider our method, which is based on his, for this application.

In this section we apply the formulas developed in $\$ 2$ to the case of binomial sampling for mites on cotton. The parameter $\theta$ represents the proportion of leaves in the field having one or more spider mites on them. We first develop the specific equations required for the various operations of the method. Next, we compute the average sample number and the operating characteristic curves, and compare them to Wilson's (1982) method and to the SPRT. Finally, we give a hypothetical example of how the method would be used in practice.

We plan to field test this method, at which point we may find that some refinement is necessary. For the initial development, however, we use the simplest statistical techniques. For example, we use conjugate families to develop the appropriate probability distributions, and we use the method of moments to estimate certain of the parameters of these distributions.

First, we give the form of the function $P(n, z$, ) used in equations (2.2) to compute the values of $l_{n}$ and $u_{n}$. We use a beta density to characterize the subjective distribution of $\mathbf{0}$. Therefore, we have

$$
\pi(\theta)=\frac{\Gamma(\gamma+\delta)}{\Gamma(\gamma) \Gamma(\delta)} \theta^{\gamma-1}(1-\theta)^{\delta-1} .
$$

Substituting equation (3.1) into (2.3) and working out the integral in equation (2.1) yields

$$
P\left(n, z_{n}\right)=\frac{\Gamma(\gamma+\delta+n)}{\Gamma\left(\gamma+z_{n}\right) \Gamma\left(\delta+n-z_{n}\right)} \sum_{k=0}^{\delta+n-z_{n}-1}(-1)^{k}\left(\begin{array}{c}
\delta+n-z_{n}-1 \\
k
\end{array}\right) \frac{T^{\gamma+z_{n}+k}}{\gamma+z_{n}+k} .
$$

In our example application, we use for the initial prior at the first sample a noninformative 
prior. There are several noninformative priors available for a parameter with a binomially distributed likelihood (Berger, 1980). These include a prior derived by reformulation arguments for which $\mathrm{y}=6=0$, one derived by the method of Box and Tiao (1973), for which $\gamma=6=\frac{1}{2}$, and the uniform prior, for which $\mathrm{y}=6=1$. We wish for simplicity to work with integral $\gamma$ and 6, and to use a proper prior [cf. equation (2.1)]; therefore, we use the uniform prior. Figure 1 shows the upper and lower stopping curves generated by solving equations (2.2) using $P(n, z$,$) as defined in equation (3.2) with \gamma=6=1$, and with error rates $\alpha=\beta=0.1$. The density used to compute these curves is the posterior $\pi(\theta \mid I$, ,) obtained from equation (2.3) with $\ell(\theta \mid I)=,\theta^{\gamma+z_{n}-1}(1-\theta)^{\delta+n-z_{n}-1}$. Also shown are the stopping curves obtained from Wilson's (1982) method for the same error rates, and those obtained from the SPRT with the same error rates. The SPRT requires that the threshold be defined in terms of two values $p_{0}$ and $p_{1}$, where $p_{0}<T<p_{1}$. For purposes of comparison we use $p_{0}=0.4$ and $p_{1}=0.6$, values commonly used in practice. We stress that the comparison of our method with the SPRT is an informal one.

We assume, based on our field data, that the growth of $\theta(t)$ follows roughly $a$ logistic pattern, i.e.,

$$
\theta(t)=f[\theta(0) ; r, t]=\frac{\theta(0)}{[1-\theta(0)] e^{-r t}+\theta(0)} .
$$

The density $\rho(r)$ is assumed to be normal with mean $\mu$ and variance $\sigma^{2}$.

The prior density $\pi_{t}(\theta)$ at a future time $t$ is computed from equation (2.5) where

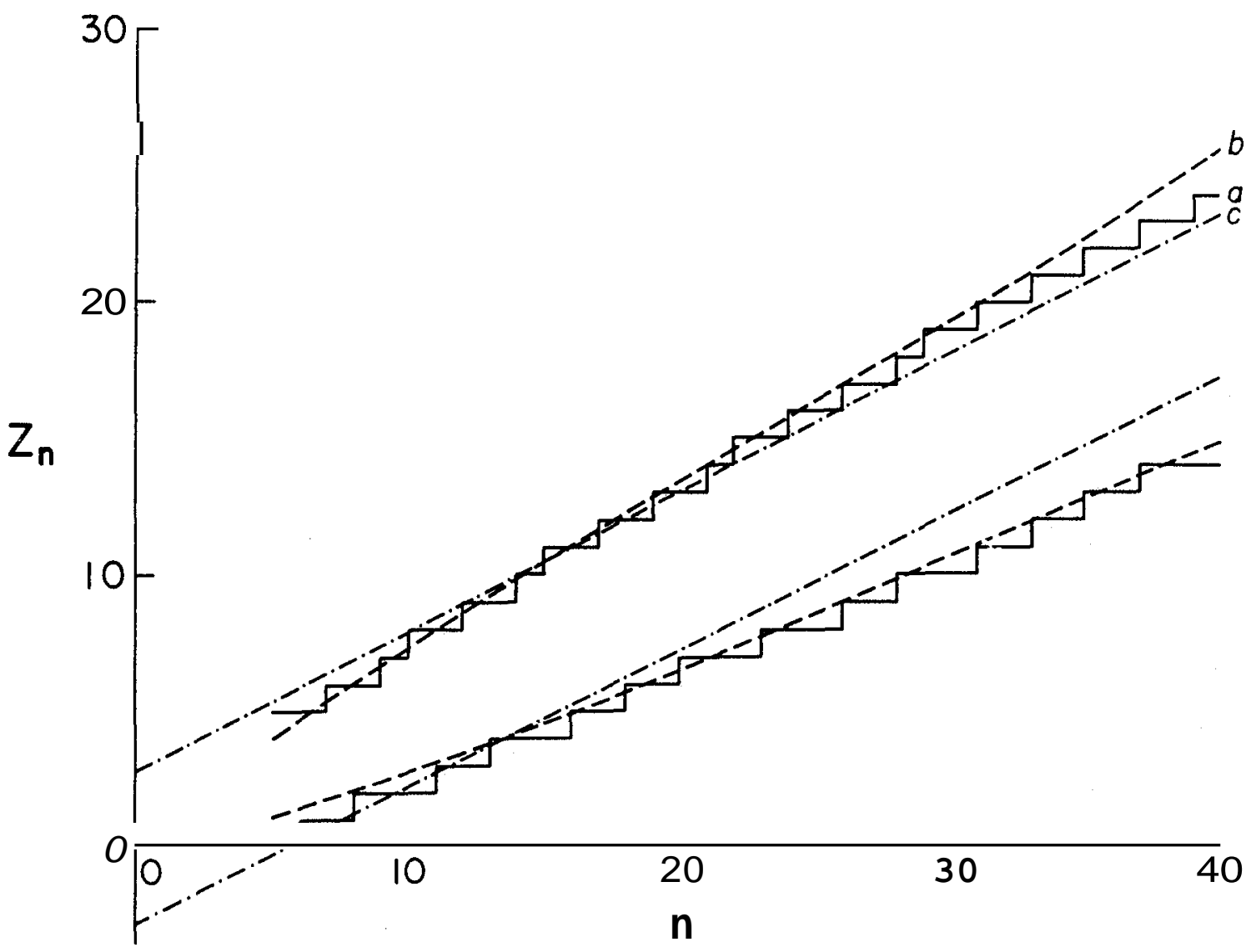

Figure 1. Stopping curves for three sequential sampling methods. Curves a represent the Bayesian method with $\gamma=\delta=1$. Curves $b$ represent Wilson's (1982) method. Curves $c$ represent the SPRT. 
$\psi(\theta ; r, t)$ is defined by equation (2.4). The function $\phi$ in this equation is given by

$$
\phi(\theta ; r, t)=\frac{\theta}{\theta^{+}(1-\theta) e^{r}} .
$$

We solve equation (2.5) numerically for $\pi_{l}(\theta)$ at a sequence of values of 8 . We then approximate this density by a beta density obtained using the method of moments. The actual density that we use at time $t$ is then obtained by rounding off the parameters $\gamma$ and 6 to the nearest integer. Figure 2 shows stopping curves for several values of the parameters $\gamma$ and 6 . We will defer comment on the figure to $\$ \mathbf{4}$.

To facilitate comparison of the various methods, we compute the average sample number (ASN) and operating characteristic $(\boldsymbol{O C})$ curves for the above scheme. This is done by recognizing that the sequential sampling procedure is a Markov chain with absorbing boundaries that move as the chain progresses. Assume that the scout always observes at least $n_{0}$ leaves and observes a maximum of $n_{1}$ (in principle, we could have $n_{0}=0$ and $\left.n_{1}=\infty\right)$. Then the average sample number is given by

$$
\operatorname{ASN}(\theta)=\sum_{n=n_{0}}^{n_{1}} n \operatorname{Pr}(N=n)
$$

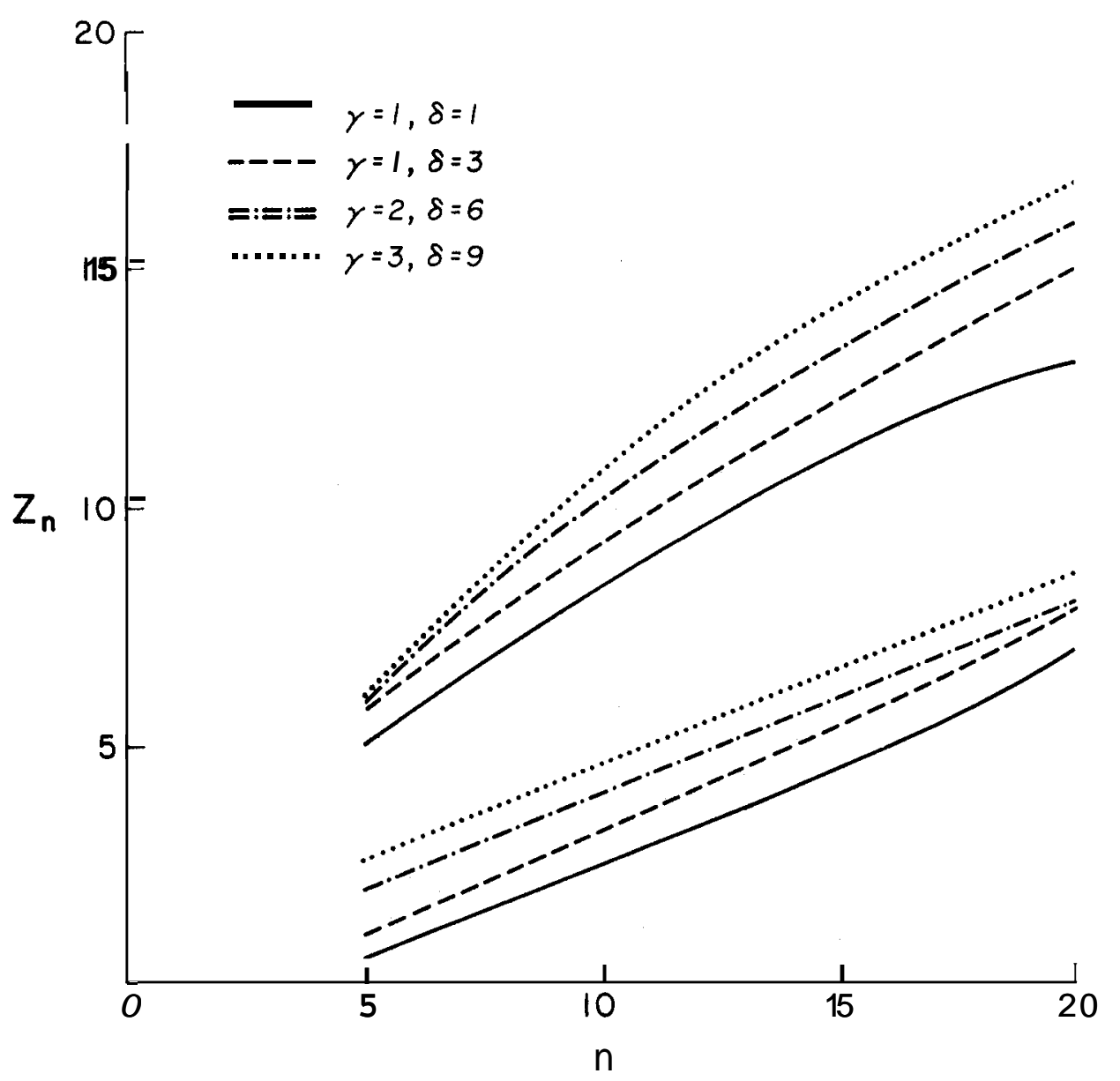

Figure 2. Schematic diagram of stopping curves for the Bayesian method for several values of the parameters $\gamma$ and $\delta$. Except for the case $y=\delta=1$, the expected value of $\theta$ in each case is 0.25 . To aid the eye, smooth curves are plotted; the actual stopping curves take on only integer values. 
Let $a_{i}(\hat{n})=\operatorname{Pr}\left(z=z_{n} \mid n=\hat{n}\right)$. Then

$$
a_{i}\left(n_{0}\right)=\left(\begin{array}{c}
n_{0} \\
i
\end{array}\right) \theta^{i}(1-\theta)^{n_{0}-i}
$$

The probability of stopping at $n_{0}$ is

$$
\operatorname{Pr}\left(N=n_{0}\right)=\sum_{i=0}^{l_{n}} a_{i}\left(n_{0}\right)+\sum_{i=u_{n}}^{n_{0}} a_{i}\left(n_{0}\right) .
$$

For any $n>n_{0}$ we have

$$
a_{i}(n)=(1-\theta) b_{i}(n-1)+\theta b_{i-1}(n-1)
$$

where

$$
b_{i}(n)= \begin{cases}a_{i}(n-1) & l_{n-1}<n-1<u_{n-1} \\ 0 & \text { otherwise }\end{cases}
$$

For $n_{0}<n<n_{1}$ we have

$$
\operatorname{Pr}(N=n)=a_{l_{n}}(n)+a_{k_{n}}(n)
$$

and for $n=n_{1}$ we have

$$
\operatorname{Pr}\left(N=n_{1}\right)=\sum_{i=I_{n}}^{u_{n}} a_{i}\left(n_{1}\right) .
$$

To test this method of computing the ASN, we compared it with the approximation given by Wald (1947, p. 99) for the ASN of the SPRT. A detailed comparison with Wald's approximation is not possible because our method of computing the ASN eliminates the event of $n$ attaining a very large value. For values of 8 near 0 or 1 , however, such events are extremely rare. For this range of values of $\theta$ the methods differed by about $\mathbf{1 0 \%}$.

The OC curve, which is the probability of deciding that the infestation is over the economic threshold given the value of 8, may be computed similarly. Equations (3.7), (3.9), and (3.10) are used to determine the probability of first crossing the upper stopping curve at a given $n$. We have

$$
\mathrm{OC}(\theta)=\sum_{i=0}^{l_{n}} a_{i}\left(n_{0}\right)+\sum_{n=n_{0}+1}^{n_{1}-1} a_{i}(n)+\sum_{i=T_{0}}^{u_{n}} a_{i}\left(n_{1}\right),
$$

where $T_{0}=n^{*} / n_{1}, n^{*}$ being the smallest integer such that $T_{0} \geqslant T$.

In actual practice, appropriate values for $n_{0}$ and $n_{1}$ for spider mites on cotton are $n_{0}=5$ and $n_{1}=40$. The lower sample limit $n_{0}$ is set greater than 1 due to the possibility of heterogeneity in the environment leading to a biased estimate of 8 . Figure $\mathbf{3}$ shows the ASN curves for our method, Wilson's (1982) method, and the SPRT. The ASN curve for the SPRT was computed by the Markov chain method for proper comparison. The thresholds for both the Bayesian and Wilson's method were fixed at 0.5. It is evident from Fig. 3 that the ASN and OC curves of the three methods are quite similar, at least for $\gamma=\delta=1$. We emphasize again that this is an informal comparison of the methods as they would be used in the field. Thus, the seeming suboptimality of the SPRT, contrary to Wald's theorem, stems from the choice of $p_{0}$ and $p_{1}$, which reflect agricultural practice. In fact, a precise mathematical comparison does have the SPRT with the lowest $p_{0}$ and $p_{1}$; note that the $\boldsymbol{O C}$ curve of the SPRT is sharper than that of the other two methods.

We conclude this section by presenting the results of an initial test of the Bayesian sampling scheme. This test consists of a simulated season in the San Joaquin Valley. The 

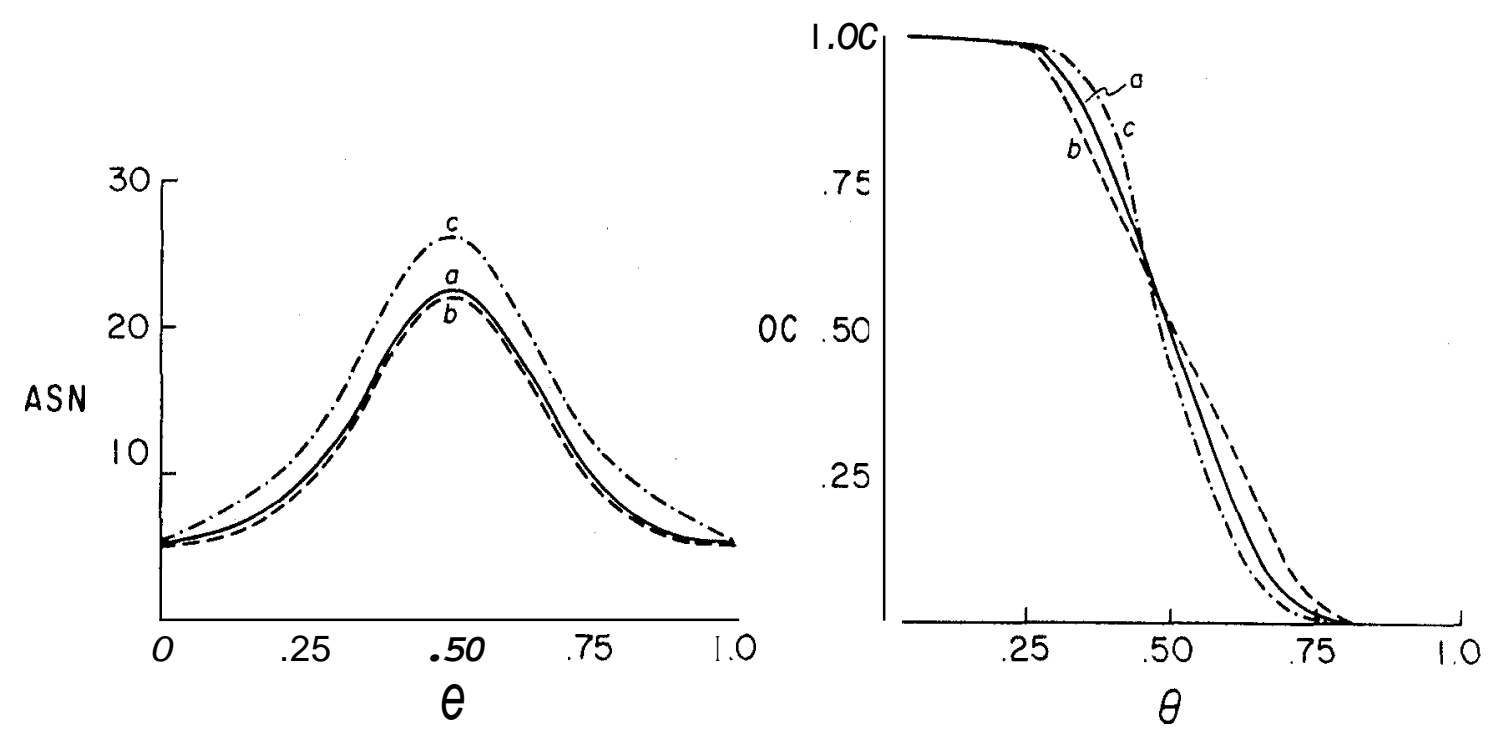

Figure 3. Average sample number (ASN) and operating characteristic (OC) curves for each of the three methods, as in Fig. 1.

sampling takes place at 100 degree-day ("D) intervals. To simulate the sampling itself we generate random values from a negative binomial distribution, using standard methods provided in Rubinstein (1981). The true value of 8, the proportion of infested leaves, is assumed to be $8=0.01$ at time $t=0$, and to follow a logistic growth curve with $r=0.01$ $\left({ }^{\circ} \mathrm{D}\right)^{-1}$. In each sequence, the stopping curves are first computed using the current values of y and 6. The sampling then takes place, stopping when the stopping curve is first crossed. The current values of $\gamma$ and $\delta$ are then replaced by $\mathrm{y}+z_{n}$ and $6+n-z_{n}$. At each stage after the first, the values of $\mu$ and $\sigma$ are then updated using equation (2.7). Next, the value of $\pi_{t}(p)$ is computed for the next value of t using equation (2.5). Finally, a forecast of future growth of the pest population is made using the same equation.

The initial values of $\gamma$ and 6 are both 1 . The starting values of $\mu$ and $\sigma$, based on data from an experimental plot at the University of California West Side Field Station in Five Points, California, are $\mu=0.0072$ and $\sigma=0.00068$. The results of the test are shown in Table 1 . With the exception of $\mathrm{t}=400^{\circ} \mathrm{D}$, for which the values of $z_{n}$ happened to be unusually large for small $n$, the sample sizes are small. The forecasts (median and tenth and ninetieth percentiles) are shown in Figure 4. These provide a reasonably good picture of what is occurring. It is likely that, based on these forecasts, in actual practice an additional sample would be taken at about $450^{\circ} \mathrm{D}$ since the population is near the economic threshold. The values in the 8 column are the true values of $\theta$ for the corresponding $t$.

Table 1

Results of a simulated season of sequential sampling

\begin{tabular}{|c|c|c|c|c|c|}
\hline$t$ & $\gamma, \delta$ & $\mathrm{n}$ & $z_{n}$ & Decision & $\theta$ \\
\hline $\begin{array}{r}0 \text { "D } \\
100 \text { "D } \\
200 \text { "D } \\
300 \text { "D } \\
400 \text { "D } \\
500 \text { "D }\end{array}$ & $\begin{aligned} 1, & 1 \\
1, & \mathbf{4} \\
1, & 6 \\
1, & 7 \\
\mathbf{4}, & 6 \\
\mathbf{2 9}, & \mathbf{2 0}\end{aligned}$ & $\begin{array}{r}5 \\
5 \\
5 \\
5 \\
40 \\
5\end{array}$ & $\begin{array}{r}0 \\
0 \\
0 \\
2 \\
17 \\
4\end{array}$ & $\begin{array}{l}\theta<T \\
\theta<T \\
\theta<T \\
\theta<T \\
\theta<T \\
\theta>T\end{array}$ & $\begin{array}{l}.01 \\
.03 \\
.07 \\
.17 \\
.36 \\
.60\end{array}$ \\
\hline
\end{tabular}



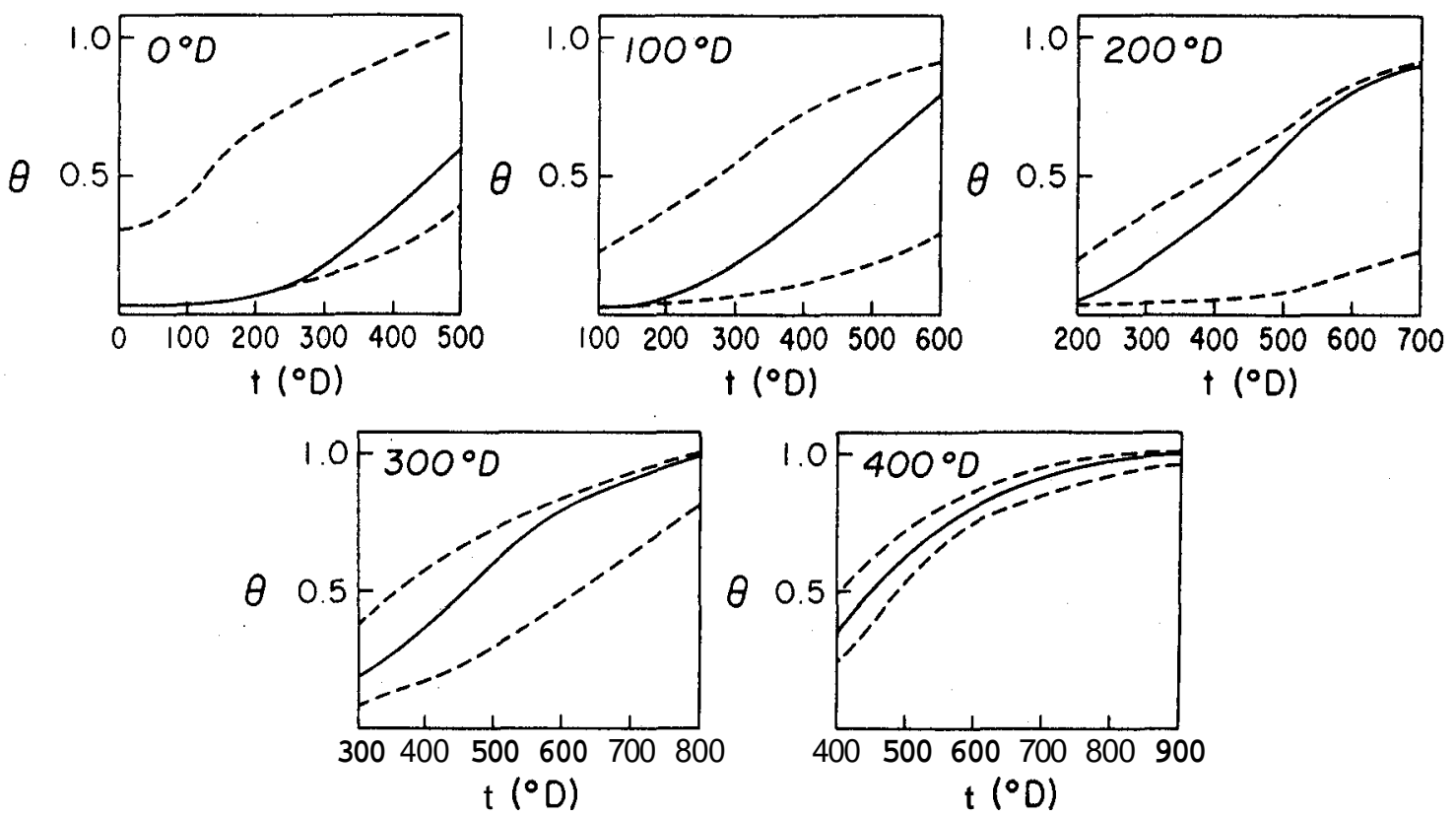

Figure 4. Eighty percent confidence intervals for projected growth of the population, forecast for $500^{\circ} \mathrm{D}$ from the time of sampling. The value at the top of each figure represents the time of sampling. Note that the prediction becomes more precise with each successive sample.

\section{Discussion}

We expected two primary dividends from the formulation of the problem of sequential estimation of arthropod populations in a Bayesian way. The first is the ability to incorporate prior information, particularly the results of previous samples, into the sampling procedure. The second is the ability to readily forecast the future growth of the population. The sampling procedure was designed to fulfill these expectations. The results of $\mathbf{\$ 3}$ indicate that, at least in principle, we have achieved our goal. The question of whether our method is practical in the field awaits further investigation. We devote this section primarily to a discussion of the test results at the end of $\S 3$.

Fig. 1 is primarily a comparison of Wilson's (1982) method with that obtained from our method using a noninformative prior. It is reassuring, although perhaps not completely surprising, that these two methods produce nearly identical results. As may be seen from the figure, the two methods produce stopping curves that tend to diverge relative to the parallel lines of the SPRT. In determining the ASN of the methods this causes the truncation of sampling at $n=\mathbf{4 0}$ to work to the disadvantage of the SPRT, since large $n$ are eliminated. Conversely, it makes the estimation methods more likely to terminate after a small number of samples. In Table 1 , with the exception of the sequence at $t=400^{\circ} \mathrm{D}$, all samples are terminated at $n=5$. It so happened that for $t=400^{\circ} \mathrm{D}$ the values of $z_{n}$ for low $\mathrm{n}$ were unusually high; in addition, the value of 8 was near the economic threshold. In any case, it is evident from Fig. 3 that the ASN and OC curves of the three methods are not greatly different.

Fig. 2 shows how the stopping curves change as $\gamma$ and $\delta$ are changed. For each of the combinations in the figure the expected value of $\pi(\theta)$ is 0.25 . It is evident from the figure that both of the stopping curves move up as the uncertainty about 8 is reduced. This is not surprising since it indicates a firmer subjective opinion that the population is, in this case, below the economic threshold.

The forecasts shown in Fig. 4 provide a good picture of what is actually going on. As 
information is accumulated about the infestation, reflected in the increase in $\gamma$ and 6 , the forecasts become sharper and sharper. An important feature of threshold-based estimation procedures such as Wilson's (1982) and ours is that they require more information precisely when it is most useful. When $\theta$ is quite far from $T$, the confidence intervals used to make the decision may be quite wide, but when $\theta$ is close to $T$ these intervals must be quite narrow. It is when 6 is close to $T$ that the most accurate information is needed, This is in part the cause of the high value of $n$ required at $t=400^{\circ} \mathrm{D}$; this high $n$ is used to sharpen considerably the subsequent forecast. Our method also has the advantage over conventional sequential analysis procedures of not requiring a second, essentially arbitrary threshold.

Some might view the ability to incorporate a forecasting scheme into the sampling scheme as the most useful aspect of our method. It is worth pointing out that, provided a reasonably good estimate of the growth rate of the population is available, the forecasting may be done independently of how the sample is taken. That is, the adjustments of $\pi_{I}(\theta)$ depend only on the values of $n$ and $z_{n}$, and not on the values used to collect them.

The sampling of mites is quite difficult because of their small size and potential abundance. Our method is appropriate without modification for other agricultural pests that, for one reason or another, are sampled binomially rather than by counting. In general, however, counting is a superior method when it is practical. In principle, our method as presented in $\$ 2$ may be applied directly to such problems. One would simply use a negative binomial density for $\pi(\theta)$. In practice, this method is difficult because the negative binomial distribution does not have a simple sufficient statistic for $\boldsymbol{k}$. We are presently developing a sampling-forecasting scheme for counting applications, and will describe it in a forthcoming paper.

\section{ACKNOWLEDGEMENT}

We are grateful to M. Mangel for some helpful discussions.

\section{RÉSUMÉ}

Nous présentons une méthode d'estimation séquentielle de l'état d'infestation d'une culture par un arthropode ravageur. La méthode basée sur la théorie statistique bayésienne, Ctablit des courbes d'arrêts en contraignant un intervalle de confiance à être placé d'un côté ou de l'autre d'un seuil économique. Les résultats d'un échantillon séquentiel sont utilisés pour modifier la distribution à priori de l'échantillon suivant en faisant l'hypothèse que l'infestation suit une certaine loi de croissance. Les paramètres de cette loi de croissance sont eux-mêmes inconnus et sont décrits par des distributions subjectives qui sont modifiées en utilisant l'échantillonnage. Un avantage majeur de notre méthode est qu'elle admet facilement I'incorporation d'un schéma de prévision. Après une description en termes généraux du développement de la méthode, nous donnons un exemple spécifique de l'application de la méthode à l'échantillonnage de "spider mites" sur coton dans la San Francisco Valley en Californie.

\section{REFERENCES}

Allen, J. D., Gonzalez, D., and Gokhale, D. V. (1972). Sequential sampling plans for the bollworm, Heliothis zea. Environmental Entomology 1, 771-780.

Anscombe, F. J. (1949). Large-sample theory of sequential estimation. Biometrika 36, 455-458.

Berger, J. O. (1980). Statistical Decision Theory. New York: Springer Verlag.

Box, G. E. P. and Tiao, G. C. (1973). Bayesian Inference in Statistical Analysis. Reading, Massachusetts: Addison-Wesley.

Iwao, S. (1975). A new method of sequential sampling to classify populations relative to a critical density. Researches in Population Ecology 16, 281-288.

Kuno, E. (1969). A new method of sequential sampling to obtain the population estimates with a fixed level of precision. Researches in Population Ecology 11, 127-136.

Onsager, J. A. (1976). The rationale of sequential sampling, with emphasis on its use in pest 
management. Technical Bulletin 1526. Washington, D.C.: Agricultural Research Service, U.S. Department of Agriculture.

Rubinstein, R. Y.(1981). Simulation and the Monte Carlo Method. New York: Wiley.

Sterling, W. L. (1976). Sequential decision plans for the management of cotton arthropods in southeast Queensland. Australian Journal of Ecology 1, 265-274.

Stern, V. M., Smith, R. F., van den Bosch, R., and Hagen, K. S. (1959). The integrated control concept. Hilgardia 29, 81-101.

Sylvester, E. S. and Cox, E. I. (1961). Sequential plans for sampling aphids on sugar beets in Kern County, California. Journal of Economic Entomology 54, 1080-1085.

Taylor, L. R. (1961). Aggregation, variance and the mean. Nature 189,732-735.

Wald, A. (1947).SequentialAnalysis. New York: Wiley. (Republished in 1973 by Dover Publications, New York.)

Waters, W. E. (1955). Sequential analysis of forest insect surveys. Forest Science 1, 68-79.

Wetherill, G. B. (1966). Sequential Methods in Statistics. London: Chapman and Hall.

Wilson, L. T. (1982). Development of an optimal monitoring program in cotton: Emphasis on spider mites and Heliothis spp. Entomophaga 27, 45-50.

Wilson, L. T. and Barnett, W. W. (1983). Degree-days: An aid in crop and pest management. California Agriculture 37, 4-7.

Wilson, L. T., Gonzalez, D., Leigh, T. F., Maggi, V., Foristiere, C., and Goodell, P. (1983). Withinplant distribution of spider mites (Acari: tetranychidae) on cotton: A developing implementable monitoring program. Environmental Entomology 12, 128-134.

Wilson, L. T. and Room, P. M. (1983). Clumping patterns of fruit and arthropods in cotton with implications for binomial sampling. Environmental Entomology 12, 50-54.

Zalom, F. G., Wilson, L. T., Hoffmann, M. P., Lange, W. H., and Weakley, C. V. (1983). Monitoring lepidopterous pest damage to processing tomatoes. California Agriculture 37, 25-26.

Received January 1984; revised June and November 1984. 